\title{
Factores de riesgos de muerte súbita cardiaca en adolescentes: una revisión sistemática
}

\author{
Risk factors for sudden cardiac death in adolescents: a systematic review
}

\author{
Zammir Rodríguez (D)
}

${ }^{1}$ Universidad Ricardo Palma, Instituto de Investigación en Ciencias Biomédicas, Perú.

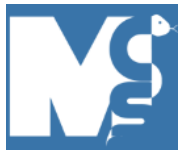

Recibido: $19 / 10 / 2021$

Revisado: 08/12/2021

Aceptado: 02/01/2022

\section{Autor correspondiente}

Zammir Rodríguez

Universidad Ricardo Palma, Instituto de Investigación en Ciencias Biomédicas, Perú. rodriguezcano98@gmail.com

\section{Conflictos de interés}

El autor declara no poseer conflictos de interés.

\section{Fuente de financiación}

El autor no recibió apoyo financiero del Gobierno Regional de Tumbes para realizar esta investigación.

Este artículo es publicado bajo una licencia de Creative Commons Reconocimiento 4.0 Internacional.

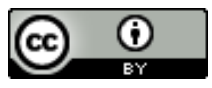

\section{RESUMEN}

Introducción: La muerte súbita cardiaca es un resultado poco común siendo en adolescentes incluso más catastrófico tanto para su entorno familiar como la sociedad, por lo cual es de vital importancia identificar los factores que pueden conllevar a una persona estar en riesgo de tener esta devastadora muerte. Objetivo: Determinar los factores de riesgo de muerte súbita cardiaca en adolescentes. Metodología: Se realizó una revisión sistemática donde se tomaron en cuenta todos los artículos en el idioma inglés y español los cuales fueron publicados desde el año 2020 hasta el año 2021, en los sitios de búsqueda PubMed y Google Scholar. Se utilizó la siguiente pregunta PEO: Población: Adolescentes, Exposición: Factores de riesgo, Outcome: Muerte súbita cardiaca. Las palabras clave fueron: "youth", en combinación con "Risk Factors" y "Sudden Cardiac Death" (utilizados en PubMed). En Google Scholar se utilizaron los siguientes términos para la búsqueda avanzada: "Adolescentes", "Muerte súbita Cardiaca" y "Factores de riesgo". Se seleccionaron los artículos publicados desde el año 2015 que cumplieran con los criterios de exclusión. Resultados: De los 13 artículos para esta revisión con respecto a la muerte súbita cardiaca en adolescentes se encontró una variedad de causas o factores de riesgo que hicieron desencadenar esta patología encontrando entre estas ciertas causantes con mayor repetición. Conclusión: La muerte súbita cardiaca tiene como principales factores de riesgo a las cardiopatías congénitas y la carga genética; los cuales debemos identificar y así poder actuar de una manera preventiva disminuyendo su incidencia de muerte.

Palabras clave: Adolescentes; Factores de riesgo; Muerte Súbita Cardiaca.

\section{ABSTRACT}

Introduction: Sudden cardiac death is an uncommon result, being in adolescents even more catastrophic for both their family environment and society, for which it is vitally important to identify the factors that can lead a person to be at risk of having this devastating death. Objective: To determine the risk factors for sudden cardiac death in adolescents. Methods: A systematic review was carried out where all the articles in English and Spanish were taken into account, which were published from 2020 to 2021, on the PubMed and Google Scholar search sites. The following PEO question was used: Population: Adolescents, Exposure: Risk factors, Outcome: Sudden cardiac death. The keywords were: "youth", in combination with "Risk Factors" and "Sudden Cardiac Death" (used in PubMed). In google scholar, the following terms were used for the advanced search: "Adolescents", "Sudden Cardiac Death" and "Risk factors". Articles published since 2015 that met the exclusion criteria were selected. Results: Of the 13 articles for this review regarding sudden cardiac death in adolescents, a variety of causes or risk factors were found that triggered this pathology, finding among these certain causes with greater repetition. Conclusion: Sudden cardiac death has as main risk factors congenital heart disease and genetic load; which we must identify and thus be able to act in a preventive manner reducing their incidence of deaths.

Keywords: Adolescents; Risk factors; Sudden Cardiac Death. 


\section{INTRODUCCIÓN}

La muerte súbita cardiovascular (MSC) continúa siendo aún en nuestros días un problema de magnitud creciente a nivel mundial. Afecta a pacientes aparentemente sanos que de forma inesperada mueren con las consecuencias que tal hecho causa a la familia y a la sociedad. Sus causas y factores de riesgo son muchas veces evitables por lo que se hace necesario la actualización de los conocimientos de esta entidad para poder realizar su prevención integral, que incluye, la modificación del estilo de vida y el diagnóstico precoz de alteraciones cardiovasculares ya que aun en nuestros días, sigue siendo la estrategia más efectiva la identificación y control de los grupos de riesgo para evitarla.

La MSC es la parada cardíaca que se produce de forma abrupta y repentina en personas que aparentemente tienen un buen estado de salud. Concluye con el fallecimiento del paciente si no recibe asistencia médica inmediata. Es considerada uno de los principales retos de la cardiología y la medicina modernas por la alta incidencia de esa problemática en el mundo por lo cual exige una mirada y un abordaje multidisciplinario (1).

Cada año, más de 400000 estadounidenses sucumben a la muerte súbita cardíaca. Aquellos que sufren de paro cardíaco pueden o no haber sido diagnosticados previamente con enfermedad cardíaca (2). La incidencia y la epidemiología de la muerte súbita están en función de la edad, los síndromes de arritmia primaria y las cardiomiopatías hereditarias son las causas predominantes en los pacientes más jóvenes, la enfermedad arterial coronaria es la etiología principal en los mayores de 35 años (3).

Hace 10-20 años no sabíamos con qué frecuencia ocurría la SCD en los jóvenes, y teníamos escasos conocimientos sobre el papel de la herencia. Hemos encontrado que la SCD corresponde al $7 \%$ de todas las muertes con una tasa de incidencia general (la más alta posible) de 2,8 por 100000 personas-año (tasa de autopsia de casos de muerte súbita del $75 \%$ ). Esta tasa de incidencia es más alta que en la región del Véneto, en los Países Bajos y en el Reino Unido (4).

La MSC es un evento poco común pero catastrófico, que puede ocurrir en neonatos y niños pequeños. Aunque se ha llevado a cabo una extensa investigación que evalúa las causas subyacentes, todavía existe un cierto grado de incertidumbre en torno a esta área. La cardiopatía congénita es una causa conocida de muerte súbita cardíaca en niños, su etiología abarca los mecanismos inducidos viralmente, la susceptibilidad genética, los factores inducidos por medicamentos y los factores maternos. Las herramientas de detección y las investigaciones que incluyen electrocardiogramas y ecocardiogramas junto con una toma de antecedentes concisa y un examen físico se pueden utilizar para identificar los posibles factores de riesgo cardiovascular de la muerte súbita (5).

Encontrar una causa genética precisa de muerte permite realizar pruebas genéticas en cascada de los miembros de la familia para identificar a aquellos que están en riesgo y facilitar la intervención temprana para prevenir otra muerte súbita. Por lo tanto, las investigaciones para definir la causa precisa de la SCD de una persona joven no solo traen un nivel de cierre para la familia, sino que también son de vital relevancia clínica (6).

Trágicamente, la muerte súbita puede ser la primera manifestación de la enfermedad en una persona y, por lo tanto, la evaluación clínica y genética de los miembros de la familia sobrevivientes forma un papel clave en el diagnóstico de la afección cardíaca hereditaria subyacente. Esto es particularmente relevante cuando se considera que la mayoría de las afecciones cardíacas hereditarias se heredan de manera autosómica dominante, lo que significa que los miembros de la familia sobrevivientes tienen una probabilidad del $50 \%$ de heredar el mismo sustrato de la enfermedad (7).

La FV suele darse en presencia de un miocardio que tiene vulnerabilidad debido a la acción de diferentes factores los cuales se incluyen un entorno genético y ambiental adecuado, y/o el incremento de la actividad simpática en relación con estrés físico o psíquico que, aunque en situaciones normales no tienen repercusión, puede ser el desencadenante de la MS en situaciones especiales (isquemia aguda), enfermedades hereditarias (miocardiopatías y canalopatías), etc. (8).

\section{METOdOLOGÍA}

En el presente trabajo de revisión sistemática se utilizaron artículos publicados en inglés y español, los cuales fueron publicados desde el año 2015 hasta el año 2021, en los sitios de búsqueda PubMed y Google Scholar. Se utilizó la siguiente pregunta PEO: Población: Adolescentes, Exposición: Factores de Riesgo, Outcome: Muerte Súbita Cardiaca. Las palabras claves fueron: "Adolescente" (Mesh), "Risk Factors" (Mesh) y "Death, Sudden, Cardiac" (Mesh). El resumen de la búsqueda avanzada fue: "Adolescent" (mh) OR Youth* (tiab) AND "Risk Factors" (mh) AND "Death, Sudden, Cardiac" (mh) OR Sudden Cardiac Death* (tiab) OR Cardiac Death* (tiab) OR Sudden Death* (tiab). Criterios de inclusión: Estudios que tengan relación con 
nuestro tema, artículos que consignen al autor y estudios realizados en los últimos 6 años. Criterios de exclusión: Estudios realizados que no tengan relación en nuestro tema, artículo que no consignen al autor y estudios realizados hace más de 6 años.

\section{RESULTADOS}

De los 1230 resultados obtenidos por PubMed se excluyeron 1200 por no cumplir con los criterios de elección debido a que no guardaban relación con el propósito del artículo y 18 por duplicación, quedando 12 artículos aptos para la revisión. De los 40 artículos hallados en SciELO, se excluyeron 38 por no cumplir con los criterios de inclusión los cuales fueron guardar relación directa con el propósito del artículo, siendo únicamente elegidos 2 artículos los cuales sí guardaban la relación deseada. Los artículos restantes se obtuvieron de Google Académico, siendo en total 14 artículos para la revisión (Figura1).

\section{FIGURA 1: BÚSQUEDA SISTEMÁTICA DE LOS ARTÍCULOS Y SU SELECCIÓN.}

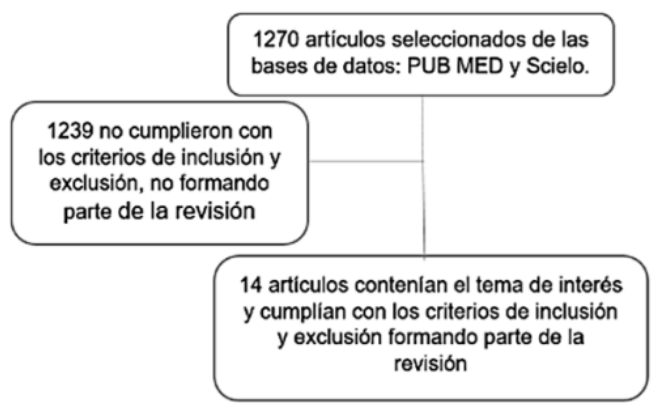

En la Tabla 1 se presentan las principales características de los 15 estudios seleccionados, apreciándose el periodo de publicación desde 2016 al 2021. Los estudios fueron desarrollados en personas de diferentes sexos, países, con un grupo etario determinado.

TABLA 1: TÍTULOS, AUTORES Y DISEÑO DE TRABAJOS DE INVESTIGACIÓN RELACIONADOS CON MUERTE CARDIACA SÚBITA

\begin{tabular}{|c|c|c|c|c|c|}
\hline AUTORES & TíTULO & AÑO & PAÍS & POBLACIÓN & DISEÑO \\
\hline $\begin{array}{c}\text { Mishra V, Zaidi S, Axiaq A, Harky } \\
\text { A. }\end{array}$ & $\begin{array}{l}\text { Sudden cardiac death in children with } \\
\text { congenital heart disease }\end{array}$ & 2020 & UK & 382 & $\begin{array}{l}\text { Observacional } \\
\text { Retrospectivo }\end{array}$ \\
\hline $\begin{array}{c}\text { Gray B, Ackerman MJ, Semsarian } \\
\text { C, Behr ER. }\end{array}$ & $\begin{array}{l}\text { Evaluation After Sudden Death in the Young: A } \\
\text { Global Approach }\end{array}$ & 2019 & UK & 115 & $\begin{array}{l}\text { Cohorte } \\
\text { retrospectiva }\end{array}$ \\
\hline $\begin{array}{l}\text { Winkel BG, Jabbari R, Tfelt- } \\
\text { Hansen J. }\end{array}$ & How to prevent SCD in the young? & 2017 & UK & & Meta análisis \\
\hline Krokhaleva Y, Vaseghi M. & $\begin{array}{l}\text { Update on prevention and treatment of } \\
\text { sudden cardiac arrest }\end{array}$ & 2019 & UK & & Meta análisis \\
\hline $\begin{array}{l}\text { Saadi S, Ben Jomaa S, Bel Hadj M, } \\
\text { Oualha D, Haj Salem N. }\end{array}$ & Sudden death in the young adult & 2020 & TÚNEZ & 137 & $\begin{array}{l}\text { Cohorte } \\
\text { retrospectiva }\end{array}$ \\
\hline $\begin{array}{c}\text { Bika Lele EC, Pepouomi MN, } \\
\text { Temfemo A, Mekoulou J, Assomo } \\
\text { Ndemba P, Mandengue SH }\end{array}$ & $\begin{array}{l}\text { Effet d'un effort intermittent d'intensité } \\
\text { variable sur la variation du QT et du risque de } \\
\text { mort subite chez des élèves camerounais }\end{array}$ & 2018 & CAMERÚN & 41 & Reporte de casos \\
\hline $\begin{array}{c}\text { Weissler-Snir A, Allan K, } \\
\text { Cunningham K, Connelly KA, Lee } \\
\text { DS, Spears DA, Rakowski H, } \\
\text { Dorian P. }\end{array}$ & $\begin{array}{c}\text { Hypertrophic Cardiomyopathy-Related } \\
\text { Sudden Cardiac Death in Young People in } \\
\text { Ontario }\end{array}$ & 2019 & CÁNADA & 44 & Reporte de Caso \\
\hline El-Assaad I, Al-Kindi SG, Aziz PF. & $\begin{array}{l}\text { Trends of Out-of-Hospital Sudden Cardiac } \\
\text { Death Among Children and Young Adults }\end{array}$ & 2017 & USA & 1452808 & $\begin{array}{l}\text { Revisión } \\
\text { Sistemática }\end{array}$ \\
\hline $\begin{array}{c}\text { Finocchiaro G, Papadakis M, } \\
\text { Dhutia H, Cole D, Behr ER, Tome } \\
\text { M, Sharma S, Sheppard MN. }\end{array}$ & $\begin{array}{c}\text { Obesity and sudden cardiac death in the } \\
\text { young: Clinical and pathological insights from } \\
\text { a large national registry }\end{array}$ & 2018 & UK & 1033 & $\begin{array}{c}\text { Revisión } \\
\text { sistemática }\end{array}$ \\
\hline
\end{tabular}


En los distintos estudios se encontró lo siguiente: Cristóbal Scmehil y colaboradores realizaron un estudio observacional prospectivo donde se analizó la demografía y la epidemiología de las muertes súbitas en atletas jóvenes utilizando el Registro Nacional de los Estados Unidos entre 1980 y 2011 . Concluyeron que dadas las investigaciones y evidencias el ECG no sería factible como método. Utilizando los informes de la autopsia, se encontró que 842 atletas tenían etiologías cardiovasculares. Entre los 842 atletas, los hombres representaron la mayoría en una proporción de 6.5: 1 hombres a mujeres. De los diagnósticos cardiovasculares específicos, la miocardiopatía hipertrófica (HCM) fue la causa más común de MSC, ocurriendo en 302 de 842 atletas ( $36 \%$ ). Algunas de las otras causas principales de MSC se identificaron como anomalías congénitas de la arteria coronaria, miocardiopatía arritmogénica del ventrículo derecho y síndrome de QT largo diagnosticado clínicamente. Los afroamericanos y otras minorías fueron los más representados ( 5 veces más que los blancos) con HCM permaneciendo como la principal causa de MSC en esa población. Los autores recomiendan el uso de eco portátil antes del ECG debido a que este no es tan rentable ni recomendado a comparación del primero el cual muestra un futuro prometedor (9).

Dijo Saadi y colaboradores realizaron un estudio de tipo cohorte retrospectiva utilizando datos de autopsia del Departamento de Medicina Legal de Monastir (Túnez). Se realizó una revisión de todas las autopsias realizadas durante 28 años; teniendo autopsias forense completas e investigaciones histológicas y toxicológicas. Se recogieron 137 casos de muerte súbita durante el periodo estudiado. La edad media de la población estudiada fue de 26,47 años. Casi el $72 \%$ de las muertes se clasificaron como muerte cardíaca, y se debieron a cardiopatía isquémica en el 32,32 \%. La muerte súbita se atribuyó a una causa pleuropulmonar en el $7,4 \%$, una causa abdominal en el $6 \%$, y de origen neurológico en el 4,5\%. Los autores llegaron a la conclusión que la MSC es con mayor frecuencia o teniendo mayor riesgo a padecerla en personas con enfermedades cardiacas insospechadas (10).

Bika Lele y colaboradores realizaron un estudio descriptivo observacional utilizando 41 estudiantes de una escuela secundaria en Doula (Camerún) con un promedio de edad de $18 \pm 2$ años. En donde cada sujeto se sometió a una prueba que comenzó con una caminata de $2 \mathrm{~km}$ seguida de una carrera de velocidad y luego una carrera de resistencia o al revés. Se registraron dos electrocardiogramas: antes del inicio de la actividad física y 5 minutos después de la última carrera. EI QT se corrigió utilizando cuatro fórmulas.
Los autores concluyeron que el riesgo de MSC aumenta significativamente después de la actividad física y el deporte, pero se necesitan más estudios sobre muestras más grandes (11).

Adaya Weissler-Snir y colaboradores realizaron un estudio descriptivo retrospectivo utilizando la base de datos de la Oficina del Forense Jefe de Ontario que abarca todas las muertes atendidas por el forense, identificamos todas las MSC relacionadas con miocardiopatía hipertrófica (HCM) en individuos con confirmación de la HCM con características macroscópicas y microscópicas típicas (MSC definida relacionada con la $\mathrm{HCM}$ ). Se identificaron cuarenta y cuatro, 3 y 6 casos de MSC definidas, probables y posibles relacionadas con HCM, respectivamente, correspondientes a tasas de incidencia anual estimadas de 0,31 por 1000 HCM persona-año. La mayoría (70 \%) de las MSC ocurrieron en individuos no diagnosticados previamente. La mayoría de las MSC ocurrieron durante el reposo (64,8 \%) o la actividad ligera (18,5\%). Los autores concluyeron que la incidencia de MSC relacionada con HCM en la población general es sustancialmente menor que la reportada anteriormente, y la mayoría de los casos ocurren en individuos no diagnosticados previamente. Las SCD se relacionan con poca frecuencia con el ejercicio (12).

Iqbai El-Assad y colaboradores realizaron un estudio descriptivo retrospectivo e obtuvieron datos demográficos y de mortalidad basados en certificados de defunción para residentes estadounidenses (19992015). Los casos de muerte súbita y muerte súbita cardíaca se recuperaron utilizando los códigos de la Clasificación Internacional de Enfermedades, 10a Revisión. Con un total 1.452 .808 sujetos de 1 a 34 años de edad murieron en los Estados Unidos, de los cuales 31.492 (2 \%) se debieron a muerte súbita cardíaca. La incidencia estimada de muerte súbita cardíaca es de 1,32 por 100.000 individuos y aumentó con la edad de 0,49 (1-10 años) a 2,76 (26-34 años). Durante el período de estudio, la incidencia de muerte súbita cardíaca disminuyó de 1,48 a 1,13 por 100.000 ( $\mathrm{P}$ < ,001). La reducción de la mortalidad se observó en todos los grupos raciales y étnicos con una magnitud variable y fue mayor en niños de 11 a 18 años. La mayoría de los niños pequeños (1-10 años) murieron de cardiopatía congénita( $n=1525,46 \%)$, mientras que los adultos jóvenes murieron con mayor frecuencia por cardiopatía isquémica. Los autores concluyeron que las tasas de mortalidad súbita cardíaca fuera del hospital disminuyeron un $24 \%$ de 1999 a 2015. Existen disparidades en la mortalidad entre los grupos de edad y los grupos raciales y étnicos, y los individuos afroamericanos no hispanos tienen las tasas de 
mortalidad más altas (13).

Gherardo Finocchiaro y colaboradores realizaron un estudio de tipo cohorte retrospectiva teniendo una población de 1033 casos los cuales se disponía información sobre el IMC, la demografía del fallecido, los antecedentes médicos, los antecedentes familiares, los síntomas cardíacos y las circunstancias de la muerte. Doscientos doce individuos (20 \%) eran obesos. En las víctimas obesas de MSC, los principales hallazgos post mortem fueron: corazón normal (síndrome de muerte arrítmica súbita), hipertrofia ventricular izquierda inexplicable y enfermedad arterial coronaria crítica(EAC). Menos frecuentes fueron la miocardiopatía hipertrófica y la miocardiopatía arritmogénica del ventrículo derecho. En comparación con las víctimas de MSC no obesas, el síndrome de muerte arrítmica súbita fue menos común, mientras que la HVI y la enfermedad arterial coronaria crítica fueron más frecuentes. La prevalencia de enfermedad arterial coronaria crítica y no crítica fue significativamente mayor en individuos obesos. Los autores concluyeron que varias enfermedades cardíacas subyacen a la MCS en la obesidad, con una prevalencia relativamente alta de síndrome de muerte arrítmica súbita, HVI idiopática y CAD. Según la literatura, el grado de hipertrofia medido por el peso del corazón y el grosor de la pared en algunos pacientes obesos con SCD es excesivo incluso después de la corrección del tamaño corporal, dejando preguntas abiertas sobre una posible relación causal entre la HVI y las arritmias fatales en este contexto. Casi uno de cada cuatro pacientes jóvenes obesos con SCD muestra un cierto grado de EAC, lo que subraya la necesidad de prevención primaria específicamente en estos individuos (14). En la Tabla 2 se presentan la importancia, fortaleza y debilidades de los 15 estudios seleccionados.

TABLA 2: IMPORTANCIA, FORTALEZA Y DEBILIDADES DE LOS 15 ESTUDIOS SELECCIONADOS

\begin{tabular}{cll}
\hline TÍTULO DEL ESTUDIO & \multicolumn{1}{c}{ IMPORTANCIA } & FORTALEZAS \\
\hline Cardiac Arrest & Es una recolección de datos con el tema Menciona las principales causas y cambio con No menciona la frecuencia no las \\
& estudiado teniendo información actualizada respecto a las edades y zona demográfica posibles \\
& y muy relevante con la muerte súbita teniendo en común el ser de aparición desencadenantes de la muerte \\
& cardiaca. & súbita cardiaca.
\end{tabular}

Sudden cardiac death in Esta revisión ha estudiado exhaustivamente La clasificación exitosa junto con la vigilancia Se debe de realizar más énfasis children with congenital heart las causas y los factores de riesgo de muerte continua del tipo de cardiopatía congénita es en la diferencia de prevención disease súbita cardíaca en niños con cardiopatía determinante crucial para mejorar la tanto primaria como secundaria coronaria y proporciona una recopilación y supervivencia de los pacientes. Las estrategias entre pacientes adultos y un resumen de la evidencia disponible hasta de prevención primaria y secundaria tienen el pediátricos. ahora que sustenta el vínculo complejo papel más importante para disminuir la entre los dos. $\quad$ incidencia de muertes súbitas cardiacas.

Update on prevention and Determinar las principales estrategias para Los desfibriladores cardioversores internos Se centra en la prevención y no treatment of sudden cardiac la prevención primaria y secundaria en siguen siendo el pilar de la prevención primaria entrega tantos datos con arrest $\quad$ pacientes con un paro cardiaco repentino y secundaria del paro cardíaco repentino. En la respecto a los factores de riesgo sin llegar a la muerte súbita cardiaca. fase aguda, la cadena cardíaca de supervivencia, asociados a la muerte súbita la reperfusión temprana y la hipotermia cardiaca. terapéutica son los pasos clave para mejorar los resultados.

$\begin{array}{ll}\text { How to prevent SCD in the } & \text { Brinda datos estadísticos, con respecto a la } \\ \text { young? } & \text { muerte súbita cardiaca y brinda múltiples } \\ & \text { factores que están asociados a una } \\ & \text { predisposición de la muerte súbita } \\ \text { cardiaca }\end{array}$
Tanto la carga genética como las cardiopatías Menciona tratamientos como congénitas son los principales factores para una posibles prevenciones sin posible muerte cardiaca súbita teniendo que mostrar efectividad con estudios actuar de una manera precoz y de forma previos. preventiva.

\begin{abstract}
Evaluation After Sudden Death in the Young: A Global Approach
\end{abstract}

Describe de una manera extensa y detallada el factor que sería el más importante para la predisposición a una muerte súbita cardiaca.

Las afecciones cardíacas hereditarias se No menciona metodología a
heredan de manera autosómica dominante, lo seguir para la prevención de
que significa que los miembros de la familia pacientes o familiares de
sobrevivientes tienen una probabilidad del $50 \%$ fallecidos por muerte súbita
de heredar el mismo sustrato de la enfermedad, cardiaca y así disminuir la
pudiendo ser candidatos a una muerte súbita incidencia de esta.
cardiaca. 
Sudden death in the young Se realizó un estudio de cohorte adult retrospectivo recogiéndose 137 casos de muerte súbita durante el periodo estudiado en autopsias del Departamento de Medicina Legal de Monastir (Túnez).
La muerte súbita en adultos jóvenes ocurre Se debe de realizar más estudios principalmente en un hombre fumador, con centrados en la evaluación de las edades comprendidas entre los 18 y los 24 años, causas no asociadas con la que ocurre en reposo, por la mañana y enfermedad cardiaca temprano en la semana. estructural.

\begin{tabular}{lrl}
\hline $\begin{array}{l}\text { Effet d'un effort } \\
\text { intermittent d'intensité }\end{array}$ & $\begin{array}{l}\text { Estudio realizado en estudiantes en la } \\
\text { tercera, primera y última clase de una } \\
\text { variable sur la variation du } \\
\text { escuela secundaria en Douala (Camerún). }\end{array}$ \\
$\begin{array}{l}\text { QT et du risque de mort } \\
\text { subite chez des élèves } \\
\text { camerounais }\end{array}$ & $\begin{array}{l}\text { Sometiéndolos a una prueba de carrera } \\
\text { luego siendo estos monitoreados por } 2\end{array}$ \\
& $\begin{array}{l}\text { EG } 2 \text { minutos antes del deporte y } 5 \\
\text { minuto después de la última carrera. }\end{array}$ \\
\hline $\begin{array}{l}\text { Cardiac screening to } \\
\text { prevent sudden death in }\end{array}$ & $\begin{array}{l}\text { Estudio realizado en atletas jóvenes } \\
\text { teniendo una población de 842, los } \\
\text { young athletes }\end{array}$ \\
& $\begin{array}{l}\text { hombres representaron la mayoría en una } \\
\text { proporción de 6.5:1 hombres a mujeres. }\end{array}$
\end{tabular}

Hypertrophic

Cardiomyopathy-Related

Sudden Cardiac Death in

Young People in Ontario

Estudio realizado utilizando a base de datos de la Oficina del Forense Jefe de Ontario que abarca todas las muertes atendidas por el forense del cual se identificaron 44,3 y 6 casos de MCS definidas, probables y posibles relacionadas con HCM, respectivamente.

Trends of Out-of-Hospital Estudio realizado con datos demográfico
Sudden Cardiac Death y de mortalidad basados en certificados de Among Children and Young defunción de residentes estadounidenses. Adults En total se cuenta con una población de 1 452808 sujetos con la finalidad de encontrar los factores de riesgo con más repetición.

Obesity and sudden cardiac Se realiza un estudio de cohorte de muerte death in the young: Clinical and pathological insights from a large national registry súbitas cardiacas ocurridas desde el 1995 al 2014 los cuales ocurrieron de una forma inesperada, este estudio solo se dio en jóvenes siendo un total de 1033 casos.
El riesgo de muerte súbita cardíaca aumenta Se necesitan más estudios sobre significativamente después del PSA. muestras más grandes.
Los ECG pueden detectar hasta el $94 \%$ de las Se necesita más investigación afecciones cardíacas más importantes, no son para determinar la rentabilidad invasivos y se consideran rentables en de esta modalidad del ECO a comparación con otras pruebas como las comparación del ECG. pruebas de Papanicolaou, la mamografía y el antígeno prostático específico (PSA)

La mayoría de los casos ocurren en individuos La incidencia de MCS relacionada no diagnosticados previamente. Las MCS se con HCM en la población general relacionan con poca frecuencia con el ejercicio. es sustancialmente menor que la reportada en estudios pasados.

Existen disparidades en la mortalidad entre los Los datos del estudio muestran grupos de edad y los grupos raciales y étnicos, y pocos casos de pacientes los individuos afroamericanos no hispanos adolescentes con muerte súbita tienen las tasas de mortalidad más altas. cardiaca.

Casi uno de cada cuatro pacientes jóvenes No se logra encontrar obesos con muerte súbita muestra algún grado condiciones específicas que sean de enfermedad arterial coronaria, lo que causantes o factores de riesgos subraya la necesidad de prevención primaria en para los pacientes jóvenes este subgrupo en particular. obesos

\section{DISCUSIÓN}

Mediante la presente revisión sistemática se observó que existen posibles factores de riesgo a presentar MSC en adolescentes, por lo cual es de vital importancia poder identificarlos para así disminuir la incidencia de esta patología que pese a no ser frecuente, en los últimos años presenta un aumento significativo de los casos repercutiendo de manera importante en la actualidad. La mayoría de los estudios fueron de tipo cohorte retrospectivo a excepción de Iqbai El-Assad que fue descriptivo retrospectivo. En casi todos los estudios se evidencia una relación positiva entre las cardiopatías congénitas y el factor genético con respecto a la MSC en adolescentes.

La MSC ocurre a menudo en adolescentes que no presentaban sintomatología alguna previa, es por eso que la carga genética de la enfermedad cardiaca es de vital importancia, debido a que hay una probabilidad de uno en dos de ser heredadas por cada pariente de primer grado. Esto nos conlleva a encontrar una causa genética precisa de muerte realizando pruebas genéticas en cascada de los miembros de la familia para así poder conocer a aquellos que están en riesgo y facilitar la intervención temprana para prevenir otra muerte súbita (6).

Tal como se evidencia en el estudio de Vaibhav Mishra (5), tanto la detección como la prevención juegan un rol importante en la disminución de casos de muerte súbita cardiaca en adolescentes. Un claro ejemplo mencionado de prevención es el que se debe realizar en el embarazo con gestantes fumadoras, debido a que el tabaquismo es un factor de riesgo significativo para las enfermedades congénitas cardiacas el cual condicionaría a los hijos tener una predisposición de una muerte súbita cardiaca. La obesidad no estaría fuertemente asociada a la MSC, teniendo solo pocos casos como evidencia que relacionan a esta con la muerte súbita cardiaca (14).

Pese a que no era el objetivo principal, es importante resaltar que como método de prevención y detección de posibles factores que podrían desencadenar una MSC en adolescentes atletas, es recomendable el uso de electrocardiograma previo a a la realización de deportes siendo esto muy útil llegando a disminuir en hasta un $90 \%$ los casos de MSC, a su vez el ecocardiograma portátil muestra un futuro 
prometedor para la prevención de la MCS (9).

Teniendo en cuanta la información previamente mencionada en las múltiples investigaciones se puede concluir que existe una relación entre la carga genética con relación a los parientes de primer grado y las cardiopatías congénitas, siendo estos los factores predisponentes a la MSC en adolescentes, siendo una patología en auge consumiendo cada vez más víctimas. También es importante resaltar la importancia de la prevención, lo que puede percutir en la disminución de adolescentes con este catastrófico final.

\section{CONTRIBUCIÓN DE LOS AUTORES}

El autor participó en la redacción del borrador, revisión crítica del manuscrito y aprobación final del mismo.

\section{REFERENCIAS}

1. Arias MEA, Arias CLM, Carbonell MMA. Muerte súbita cardiovascular. Rev Científica Estud UNIMED. 2020;2(1):143-56. URL.

2. Patel K, Hipskind JE. Cardiac Arrest. En: StatPearls. Treasure Island (FL): StatPearls Publishing; 2021. URL.

3. Krokhaleva $Y$, Vaseghi $M$. Update on prevention and treatment of sudden cardiac arrest. Trends Cardiovasc Med. 2019;29(7):394-400. https://doi.org/10.1016/j.tcm.2018.11.002

4. Winkel BG, Jabbari R, Tfelt-Hansen J. How to prevent SCD in the young? Int J Cardiol. 2017;237:6-9. https://doi.org/10.1016/j.ijcard.2017.03.083

5. Mishra V, Zaidi S, Axiaq A, Harky A. Sudden cardiac death in children with congenital heart disease: a critical review of the literature. Cardiol Young. 2020;30(11):1559-65. https://doi.org/10.1017/S1047951120003613
6. Bagnall RD, Singer ES, Tfelt-Hansen J. Sudden Cardiac Death in the Young. Heart Lung Circ. 2020;29(4):498-504. https://doi.org/10.7861/clinmedicine.18-2s-s17

7. Gray B, Ackerman MJ, Semsarian C, Behr ER. Evaluation After Sudden Death in the Young: A Global Approach. Circ Arrhythm Electrophysiol. 2019;12(8). https://doi.org/10.1161/CIRCEP.119.007453

8. Bayés de Luna A, Elosua R. Muerte súbita. Rev Esp Cardiol. 2012;65(11):1039-52.

https://doi.org/10.1016/j.recesp.2012.03.032

9. Schmehil C, Malhotra D, Patel DR. Cardiac screening to prevent sudden death in young athletes. Transl Pediatr. 2017;6(3):199-206.

https://dx.doi.org/10.21037\%2Ftp.2017.05.04

10. Saadi S, Ben Jomaa S, Bel Hadj M, Oualha D, Haj Salem N. Sudden death in the young adult: a Tunisian autopsy-based series. BMC Public Health. 2020;20(1):1915. https://doi.org/10.1186/s12889-020-10012-z

11. Bika Lele EC, Pepouomi MN, Temfemo A, Mekoulou J, Assomo Ndemba P, Mandengue SH. Effet d'un effort intermittent d'intensité variable sur la variation du QT et du risque de mort subite chez des élèves camerounais. Ann Cardiol Angéiologie. 2018;67(1):48-53. https://doi.org/10.1016/j.ancard.2017.03.003

12. Weissler-Snir A, Allan K, Cunningham K, Connelly KA, Lee DS, Spears DA, et al. Hypertrophic CardiomyopathyRelated Sudden Cardiac Death in Young People in Ontario. Circulation. 2019;140(21):1706-16. https://doi.org/10.1161/CIRCULATIONAHA.119.040271

13. El-Assaad I, Al-Kindi SG, Aziz PF. Trends of Out-of-Hospital Sudden Cardiac Death Among Children and Young Adults. Pediatrics. 2017;140(6):e20171438. https://doi.org/10.1542/peds.2017-1438

14. Finocchiaro G, Papadakis M, Dhutia H, Cole D, Behr ER, Tome $\mathrm{M}$, et al. Obesity and sudden cardiac death in the young: Clinical and pathological insights from a large national registry. Eur J Prev Cardiol. 2018;25(4):395-401. https://doi.org/10.1177/2047487317751291 